DOI: $10.3901 / J M E .2020 .04 .095$

\title{
高速离心永回流漩涡及空化特性
}

\author{
宋文武石建伟魏立超胡帅罗旭陈建旭 \\ (西华大学能源与动力工程学院 成都 610039)
}

\begin{abstract}
摘要: 基于高速离心原的比转速十分高, 通常高速离心原的进口处和内部是回流漩浴和空化空蚀等现象的高发区域。孔板等 空化抑制装置是否能针对回流漩涡起一定的抑制作用的研究还比较少。采用对诱导轮的前置空化抑制装置进行优化, 共设计 了改变装置前、后倾角等几何参数的 25 种方案进行数值模拟计算分析。优选出水力性能最好的 5 种方案并进行了回流漩浴 范围和强度的分。其中着重研究了 $\alpha$ 角度的改变是否会影响相关的的回流速度和压力。同时对高速离心原诱导轮、叶轮内部 空化发生及发展的影响分析。最终确定了 $-10^{\circ} / 20^{\circ}$ 和 $-20^{\circ} / 10^{\circ}$ 的两个最优方案, 在对回流漩浴的强度和影响范围方面表现均优 于其他方案; 气相体积分数在诱导轮和叶轮的内部分布面积很少, 强度较低; 设计工况下, 相较于 $0 \% 0^{\circ}$ 方案, 泵的效率约提 高了 $2 \%$, 更好地抑制回流漩㑂并提高了高速泵的抗空化性能, 为高速离心百内部空化的优化设计提供一定的参考意义。
\end{abstract} 关键词: 高速离心砅; 回流漩浴; 空化抑制装置; 倾角 中图分类号: $\mathrm{TH} 311$

\section{Analysis of Backflow Vortex and Cavitation of High Speed Centrifugal Pump}

\author{
SONG Wenwu SHI Jianwei WEI Lichao HU Shuai LUO Xu CHEN Jianxu
}

(School of Energy and Power Engineering, Xihua University, Chengdu 610039)

\begin{abstract}
The high specific speed of the high-speed centrifugal pump is very high. Therefore, the inlet and the inside of the high-speed centrifugal pump are usually high-incidence areas such as recirculation vortex and cavitation cavitation. As far as the current research status is concerned, whether the cavitation suppression device such as the orifice plate can suppress the reflux vortex is relatively rare. The pre-cavitation suppression device for the induction wheel is optimized. A total of 25 schemes for changing the geometric parameters such as the front and rear inclination angles of the device are designed for numerical simulation analysis. Based on this, it is preferable to select the five schemes with the best hydraulic performance and to carry out the fraction of the reflux vortex and the intensity. It focuses on whether the change in the alpha angle affects the associated reflow rate and pressure. At the same time, the influence of the high-speed centrifugal pump induction wheel and the internal cavitation occurrence and development of the impeller is analyzed. Finally, two optimal schemes of $-10^{\circ} / 20^{\circ}$ and $-20^{\circ} / 10^{\circ}$ are determined, which are superior to other schemes in terms of the strength and influence range of the reflux vortex; the gas phase volume fraction is inside the induction wheel and the impeller. The distribution area is small and the strength is low. Under the design condition, the efficiency of the pump is increased by about $2 \%$ compared with the $0 \% 0^{\circ}$ scheme, which better suppresses the reflux vortex and improves the anti-cavitation performance of the high-speed pump. It provides a certain reference for the optimization design of internal cavitation of high-speed centrifugal pump.
\end{abstract}

Key words: high-speed centrifugal pump; hackflow vortex; havitation suppression device; dip angle

\section{0 前言}

离心泵运行不稳定的主要影响因素是叶轮流道 内的射流、回流漩浴以及叶轮中的二次流等不良现 象。其中对离心原稳定性影响最大的是回流漩浴, 因为它会损耗很大能量，使离心泵效率下降，直接

20190309 收到初稿, 20190926 收到修改稿
导致水葲机组在小流量工况区运行不稳定。朱祖超 等 ${ }^{[1]}$ 认为, 叶轮旋转产生的离心力导致叶轮进口内 缘处压力更低, 形成的压差促使液流从叶片进口外 缘倒流回内缘, 回到主流。当液流回到主流时, 将 带动主流旋转形成回流漩涡。低比转速高速离心原 叶轮流道相比其他种类离心泵更加狭长, 故而在诱 导轮进口处更易产生回流漩浴的现象 ${ }^{[2]}$ 。同时, 回 流漩浴也会导致压力脉动, 加剧水百内部的空化。 空化对原的内部影响很大，严重的空化将会损坏泵 
的内部结构 ${ }^{[3-6]}$ 。黄建德等 ${ }^{[7]}$ 研究了常规叶片数的闭 式叶轮的相关问题, 采用紊流边界层计算和准三元 流场计算的方法对前盖板进行了分析, 对百进口处 回流发生点位置进行预测。带诱导轮的高速离心原 虽然对叶轮有良好的抗空蚀性能, 但其回流漩浴的 强度会有所增加, 严重影响高速泵的运行。QIU 等 ${ }^{[8]}$ 认为诱导轮回流漩浴和空蚀现象是密切相关 的, 随空蚀现象的加剧, 扬程会先升高至一个值后 突然下降。KIRIS 等 ${ }^{[9-10]}$ 对液体火箭发动机浴轮洜 进行了数值模拟分析, 发现诱导轮是引起回流漩浴 的主要原因, 管道内液流的二次流和回流受诱导轮 影响最为严重。YAMANISHI 等 ${ }^{[11-12]}$ 通过采用大浴 模拟的方法, 对诱导轮进口段回流单独进行了数值 模拟分析, 得到了相关位置回流漩浴的相关特征。 SINGHAL 等 ${ }^{[13]}$ 进行了对 NACA66 翼型的空化研 究, 所得结果与试验结果高度一致。ADRIAN 等 ${ }^{[14]}$ 基于 SINGHAL 等提出的全空化模型, 利用 FLUENT 软件研究了大容量离心原的空化性能, 得 到了较高精度的初生空化系数值。RAFAEL 等 ${ }^{[15]}$ 发 现轴流式诱导轮的流动随空化的变化而变化, 叶片 前缘附近区域容易出现空化, 主要与轴流式诱导轮 的流量、吸入口压力和工作条件有关。

综上所述, 回流漩浴对高速泵的内部流动和空 化的影响是显而易见的, 甚至可以说是十分严重的, 为了能够更好地抑制回流漩浴, 同时有效的提高高 速原的抗空蚀性能, 本文提出一种抑制回流漩涡的 装置, 即在诱导轮前装设前后不同倾角的孔板, 经 过大量方案的对比分析, 最终找到了可以抑制泵进 口回流漩浴的方法，效果非常明显。

\section{1 高速离心泵进口回流机理}

国内外很多学者对回流漩浴的特性进行了相 关的研究, FRASER ${ }^{[16]}$ 研究发现回流现象的产生原 因主要可以归结为, 在叶轮直径和流量一定时, 随 着流量变化而变化的动扬程将会超过始终不变的 静扬程, 此时扬程流量曲线上某些点的压力梯度将 会发生反向, 进而导致液流的流动方向相反, 产生 回流。

基于欧拉方程及相对运动的伯努利方程

$$
\begin{gathered}
\frac{\mathrm{d} v}{\mathrm{~d} t}=-\frac{1}{\rho} \frac{\partial p}{\partial n} \\
\frac{p}{\rho}+\frac{\omega^{2}-u^{2}}{2}=\text { 常数 }
\end{gathered}
$$

通过一系列的变换及沿等势线方向求偏导, 再 将两个方程进行合并, 最后得到回流方程为

$$
\frac{\mathrm{d} \omega}{\mathrm{d} r}+\frac{\omega}{r}=2 \omega
$$

对式(3)积分后得 $\omega r=\omega r^{2}+K$, 并推导出

$$
K=r_{0}\left(\omega_{0}-u_{0}\right)
$$

式中相对速度为 $\omega_{0}$, 圆周速度为 $u_{0}$ 。当偏离额定工 况运行时, 即部分负荷工况时, 相对速度回减小, 极有可能小于圆周速度的情况, $K$ 出现了负值, 即 液流出现了倒流, 从而产生了回流的现象。

通过研究回流的机理, 可以得知回流旋浴是靠 近叶片外缘的液流倒至进口处造成的。为了抑制回 流旋浴, OSHIMA ${ }^{[17]}$ 提出一个优化方案, 就是把孔 板装设在诱导轮进口之前, 通过试验分析可知, 孔 板的存在确可以很好的削弱诱导轮前缘回流漩浴 的产生, 同时回流漩浴对主流的影响也随之减小, 高速离心泵的空蚀特性得到改善 ${ }^{[18-19]}$ 。但是, 这种 单一形状的孔板装置, 是否是最优的呢? 孔板几何 形状及参数国内外学者均为进行研究 ${ }^{[16]}$, 因此, 本 文通过孔板基本几何参数分析, 和大量的对比数值 模拟研究, 最后确定了新型的孔板回流和空化抑制 装置。

\section{2 进口空化抑制装置优化设计}

在空化抑制装置设置在高速离心原进口的尾 部, 液流会在进口管中以轴向速度倒流, 使液流速 度呈相反的方向，随之液流又回到主流，从而达到 抑制回流漩涡的效果。在诱导轮进口与孔板间距 L 确定的情况下, 为了优化孔板形式以达到最佳的空 化抑制效果, 本文提出改变孔板的前后倾角来控制 进口段的回流漩浴大小的方案, 如图 1 所示。

即孔板厚度 $B=20 \mathrm{~mm}$, 孔板内径 $D_{0}=1.5 R$, 孔 板与诱导轮进口之间的距离为 $L=1.15 R^{[13]}$ 。孔板的 前倾角设置为 $\alpha$, 后倾角设置为 $\beta$ (图 2)。因为孔板 厚度 $B$ 为定值, 所以孔板的前倾角 $\alpha$ 和后倾角 $\beta$ 也 有一定的数值范围。由于两个倾角组合方案很多, 本文孔板前倾角 $\alpha$ 选取 5 个角度分别为 $-20^{\circ}$ 、 $-10^{\circ} 、 0^{\circ} 、 10^{\circ} 、 20^{\circ}$; 孔板后倾角 $\beta$ 选取 5 个角度分别为 $-20^{\circ} 、-10^{\circ} 、 0^{\circ} 、 10^{\circ} 、 20^{\circ}$ 。 把 $\alpha 、 \beta$ 进行两两组合组合, 共计 25 种方案。本 文经过大量的数值模拟, 基于方案对水力性能的 影响程度, 优选了五个方案进行分析, 分别为 $0^{\circ} / 0^{\circ}$ (之后用 $\alpha / \beta$ 表示每个方案中 $\alpha$ 与 $\beta$ 的角度选 取), $20^{\circ} / 20^{\circ} 、 10^{\circ} / 0^{\circ} 、 0^{\circ} / 0^{\circ} 、-10^{\circ} / 20^{\circ}$ 和 $-20^{\circ} / 10^{\circ}$ 的五个方案。

孔板示意图高速离心泵的计算模型分 5 个部 分组成(图 3), 分别为进口段、诱导轮、叶轮、蜗 壳以及出口段。其基本设计参数如表 1 所示。 

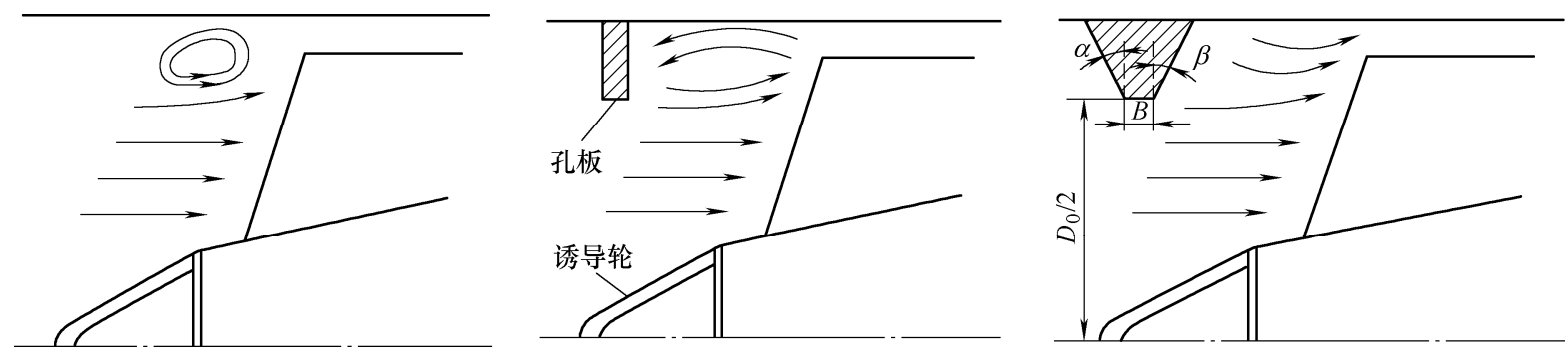

图 1 诱导轮进口处回流现象示意图

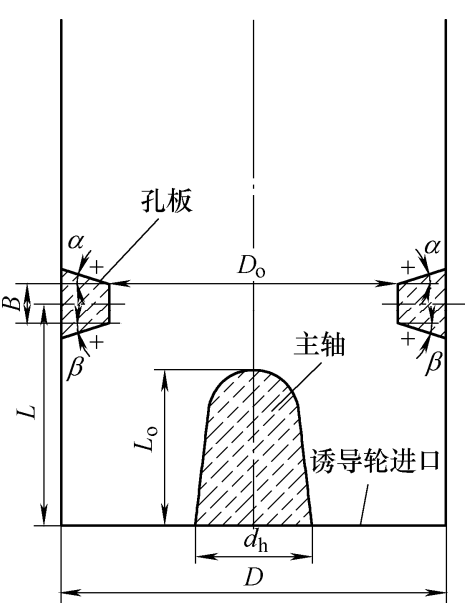

图 2 孔板示意图

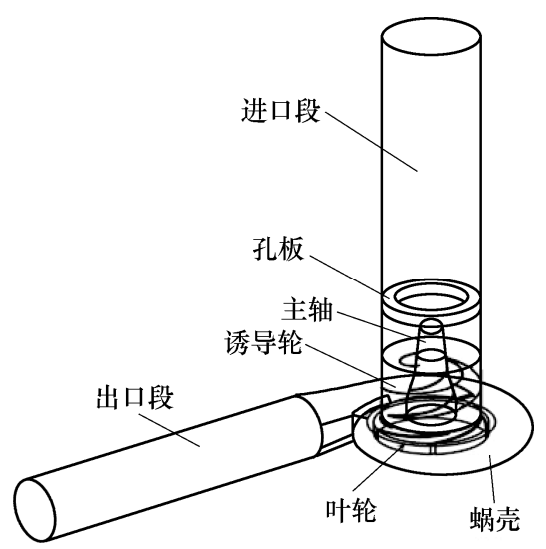

图 3 高速泵三维模型图
表 1 高速离心洜基本参数

\begin{tabular}{cc}
\hline 参数 & 数值 \\
\hline 流量 $Q /\left(\mathrm{m}^{3} / \mathrm{h}\right)$ & 662 \\
扬程 $H / \mathrm{m}$ & 620 \\
转速 $n /(\mathrm{r} / \mathrm{min})$ & 13000 \\
诱导轮 & 叶轮 \\
叶片数 $Z$ & 2 \\
叶片数 $Z_{0}$ & 5 \\
外直径 $D_{1} / \mathrm{mm}$ & 174 \\
进口内直径 $D_{2} / \mathrm{mm}$ & 52 \\
出口内直径 $D_{3} / \mathrm{mm}$ & 90.7 \\
进口直径 $D_{4} / \mathrm{mm}$ & 174 \\
出口直径 $D_{5} / \mathrm{mm}$ & 200 \\
出口宽度 $B_{0} / \mathrm{mm}$ & 19.8 \\
\hline
\end{tabular}

湍流模型采 RNG $k-\varepsilon$ 模型, 并利用 Ansys-CFX 软件分别对高速洜不加孔板和 25 种不同倾角的孔板 模型进行了分析。壁面边界条件设置为无滑移边界条 件，进口边界条件采用总压设置，出口边界条件采用 质量流速设置。对压力项采用二阶中心差分格式，其 他项采用采用二阶迎风差分格式。残差设置为 $10^{-4}$ 。

在高速离心泵的进口段截取一个沿轴向平面, 用 以研究其内部回流漩浴的变化情况, 如图 4 所示, 在 不加孔板时, 进口段轴向漩涡现象严重, 诱导轮进口 的圆周周围存在两个大漩浴, 大漩浴的存在严重堵塞 了诱导轮进口处的液流流动, 这也是泵水力性能下降 的重要原因。在加孔板之后, 回流漩涡被控制在诱导
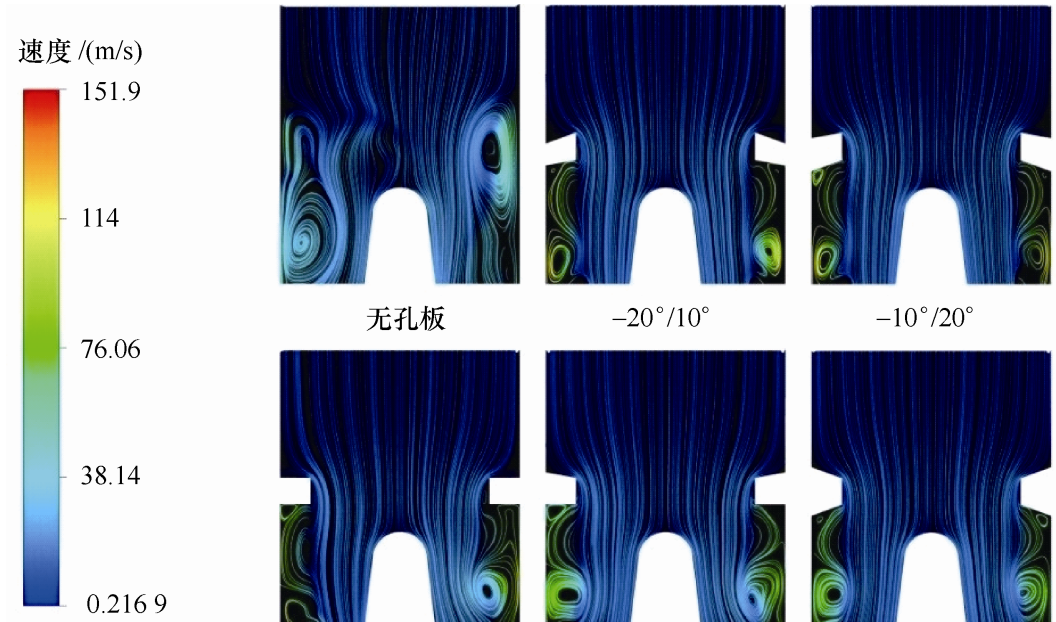

无孔板

$-20^{\circ} / 10^{\circ}$

$-10^{\circ} / 20^{\circ}$
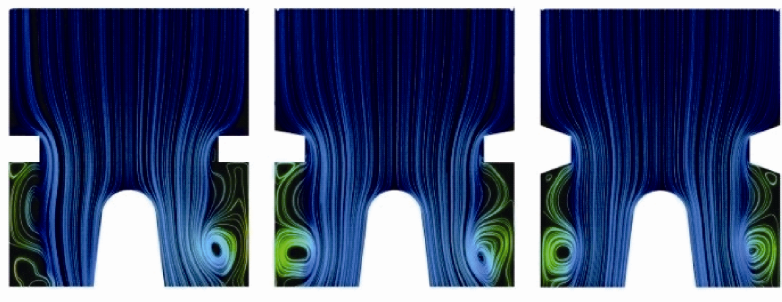

$0^{\circ} / 0^{\circ}$

$10^{\circ} / 0^{\circ}$

$20^{\circ} / 20^{\circ}$

图 4 轴向截面示意图 
轮进口与孔板之间, 进口圆周上存在两个小漩浴, 因 此受回流漩浴影响的范围大大缩小。由此可知孔板对 于抑制回流漩浴的影响范围有着不同的效果。

方案 $10^{\circ} / 0^{\circ}$ 和方案 $20^{\circ} / 20^{\circ}$ 对明显漩浴和 主流线的影响主要是使明显漩涡出现在诱导轮进口 两侧和使主流区域能正常无漩浴流动。就漩浴的强 度和影响范围来看, $-10 \% 20^{\circ}$ 和 $-20 \% 10^{\circ}$ 这两种方 案比其他三种方案要好, 浴旋的大小也比其他方案 更好, 当 $\alpha$ 角为负时, 对于回流浴的控制效果明显 优于正角。

两条监测线 $A$ 和 $B$ 布置在回流区域的轴向位置 和诱导轮入口的径向位置处(图 5), 并且在该处测量 流体的轴向速度 ( $Z$ 方向), 监测点到零点的距离记录 为 $L$, 入口段半径记录为 $R$ 。每条直线设置 50 个监 测点。

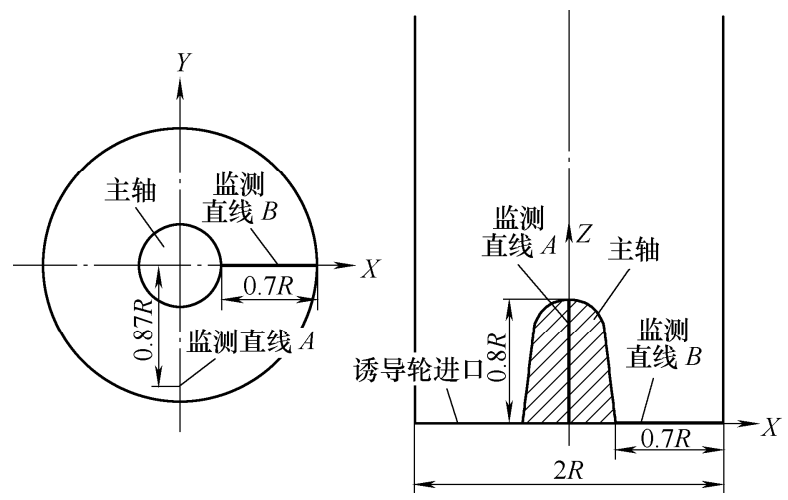

图 5 监测直线布置图

监测直线位置如图 5 所示, 轴向向下 $(Z$ 的负方 向)是主流的方向。从图 6 可以看出, 在无孔板的情 况下, 监测线 $A$ 上的轴向速度是正的, 表明进入的 流量与轴向速度相反, 也就是说, 都是回流速度, 回流漩浴在无孔板下十分严重。通过加入孔板使轴 流速度大部分与主流方向一致来达到减小主流被回 流漩涡影响的目的。对比各个孔板安放方案, 部分 方案还有较小的回流区域, 如 $0^{\circ} / 0^{\circ} 、 10^{\circ} / 0^{\circ}$

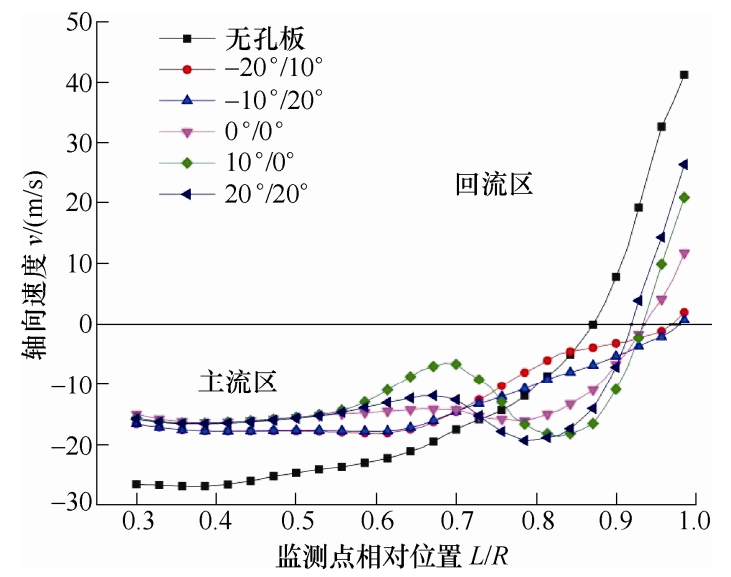

图 6 监测直线 $A$ 轴向速度
和 $20^{\circ} / 20^{\circ}$ 方案, 方案 $-10^{\circ} / 20^{\circ}$ 和 $-20^{\circ} / 10^{\circ}$ 对逆流有良好的控制作用, 因为这两种方案的回流 的轴向速度与主流一致, 并以相反的方向返回主流 区，并以相反的方向返回主流区。

观察监测线 $B$, 从图 7 中可以看出在诱导轮入口 的外边缘是回流在循环的主要区域，此处回流和主 流有相反的轴向速度。在添加孔之后, 回流区域和 具有相反轴向速度的主流明显减小, 而未添加孔板 的方案回流区域显着更大, 表明孔板能减小回流区 域。对比各个方案, 能发现方案 $-10^{\circ} / 20^{\circ}$ 和 $-20^{\circ} / 10^{\circ}$ 的控制效果最为显著, 其他三个方案对减小回流区 域有一定的作用, 但效果不如方案 $-10^{\circ} / 20^{\circ}$ 和 $-20^{\circ} /$ $10^{\circ}$, 监测线 $B$ 上的回流速度已经完全消失，表明方 案 $-10^{\circ} / 20^{\circ}$ 和 $-20^{\circ} / 10^{\circ}$ 对液流的逆流控制效果最佳。

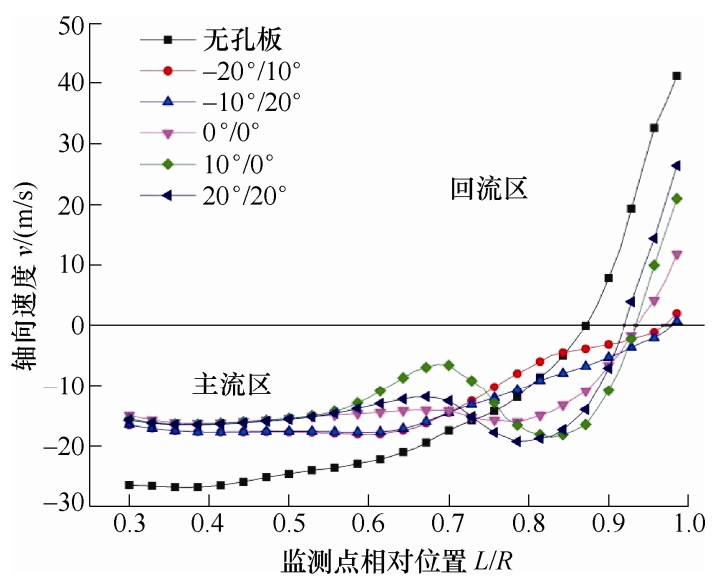

图 7 监测直线 $B$ 轴向速度

为了研究孔板倾角 $\alpha$ 对高速离心洜内部流场的 影响, 分析诱导轮进口监测直线 $B$ 处的压力和速度 分布情况, 确定 $\beta$ 值为一个定值 $0^{\circ}$, 改变 $\alpha$ 角的 度数查看其对诱导轮速度和压力的影响。监测诱导 轮入口处的流体的轴向速度和静压, 在该图中, 从 监测点到零点的距离记录为 $L$, 入口部分的半径表 示为 $R$, 并且为每条直线设置 50 个监测点。图 8 是 处理上述结果后得到的轴向速度分布图, 由此可见，

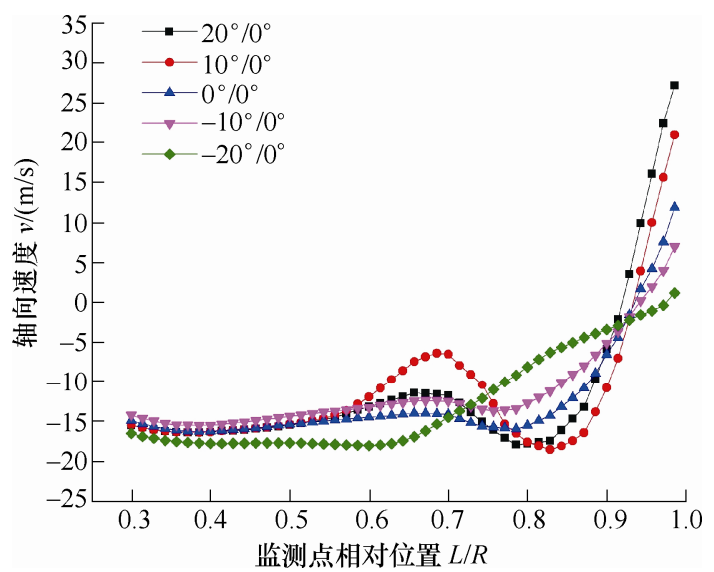

图 8 监测直线 $B$ 轴向速度 
回流主要集中在诱导轮进口的外缘处, 在诱导轮进 口外缘处的速度 $-20^{\circ} / 0^{\circ}$ 速度最小, 对回流的控制 效果也最好; $20^{\circ} 10^{\circ}$ 的速度最大, 回流的区域也 比较大。随着 $\alpha$ 角度的增大速度逐渐增大。靠近诱 导轮轮觳处速度变化规律并不太明显, 但 $-10^{\circ} / 0^{\circ}$ 的速度要小于另外几个方案。从轴向速度可看出, $\alpha$ 值对诱导轮进口处的速度大小有一定的影响。

同样对图 9 所示的压力分布规律来看, 诱导轮 进口外缘处的速度 $-20^{\circ} / 0^{\circ}$ 速度最小, 此处的压力 最大, 对于减小诱导轮进口前缘处的低压区有一定 的改善作用。因此, 在诱导轮的外缘处, $\alpha$ 为负值 时, 对于提高此处的压力是有很大的作用的。

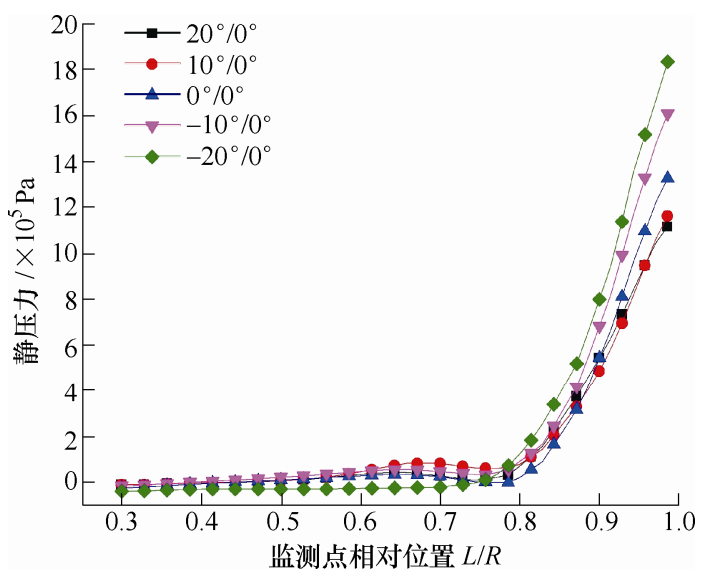

图 9 监测直线 $B$ 静压力分布

综上分析, 可以看出 $\alpha$ 为负值时, 对提高诱导轮 进口外缘的压力有一定的作用, 从而改善了诱导轮叶 轮进口前缘的低压区, 提高了诱导轮抗空化的性能。

\section{3 空化特性分析}

基于每个人对空蚀空化的理解逐渐深入而提出 了各种适应各种流动状态的空化模型。但大多数空 化模型是基于 Rayleigh-Plesset 方程的改进的空化模 型。在本文中, 使用混合模型, 混合模型是简化的 多相流模型, 两相混合物用作整个研究机构, 混合 模型表示如下。

连续性方程

$$
\frac{\partial}{\partial t} \rho_{m}+\nabla \cdot \rho_{m} v_{m}^{w}=n
$$

质量平均速度为

$$
v_{m}^{w}=\frac{\sum_{k=1}^{n} a_{k} \rho_{k} v_{k}^{w}}{\rho_{m}}
$$

式中, $a_{k}$ 为第 $k$ 相的体积份额, 混合密度 $\rho_{m}=\sum_{1}^{n} a_{k} \rho_{k}, n$ 为空化效应产生的质量传递, $\rho_{k}$ 为
第 $k$ 的密度。

动量方程

$$
\begin{gathered}
\frac{\partial}{\partial t} \rho_{m} \boldsymbol{v}+\nabla \cdot \rho_{m} \boldsymbol{v}_{m}=-\nabla P+\nabla \cdot\left[\mu_{m}\left(\nabla \boldsymbol{v}_{m}+\nabla \boldsymbol{v}_{m}\right)\right]+ \\
\rho_{m} g+F+\nabla \cdot\left(\sum_{k=1}^{n} a_{k} \rho_{k} \boldsymbol{v}_{d r \cdot k} \boldsymbol{v}_{d r . k}\right)
\end{gathered}
$$

式中, $n$ 为相数, 混合黏性系数 $\mu_{m}=\sum_{k=1}^{n} a_{k} \mu_{k}, F$ 为 体积力, 第二项 $k$ 的漂移速

$$
\boldsymbol{v}_{d r . k}=\boldsymbol{v}_{k}-\boldsymbol{v}_{m}
$$

\section{1 诱导轮空化特性分析}

利用后处理软件对每个方案的诱导轮在设计流 量工况下的压力面和吸力面及流道内部的气相体积 分数进行处理分析。

从图 10 的诱导轮吸力面汽相体积数分布图可 以明显看出, 在不添加孔板的情况下, 诱导轮的空 化非常严重, 汽相体积分数布满叶片大半个区域, 并逐渐从叶轮入口到出口逐渐衰减。其空化分布与 单相低压区的分析一致。



图 10 诱导轮吸力面汽相体积分数分布图

加入孔板后, 空化现象明显改善, 尤其是 $-20^{\circ} / 10^{\circ}$ 和 $-10^{\circ} / 20^{\circ}$ 两种方案, 其对空化现象的 抑制作用最明显, 效果最好。仅在诱导轮进口前缘有 气相体积数分布出现。在入口的前缘, 空泡的面积 远小于其他三个选项。其他三种方案的气相体积分 数主要分布在叶片的外缘, 占据叶轮一半以上的区 域, 相对于 $-20^{\circ} / 10^{\circ}$ 和 $-10^{\circ} / 20^{\circ}$ 两个方案比较混 乱, 变化比较剧烈, 且空泡的分布没有明显的规律性。

结合图 11 分析各方案压力面空化分布可以发 现, 不添加孔板的方案中, 诱导轮的压力面几乎没 有气相体积分数的分布, 但吸力面空化非常严重。 添加孔板的方案中, $-20^{\circ} / 10^{\circ}$ 和 $-10^{\circ} / 20^{\circ}$ 的两 种方案在诱导轮吸力面上的情况与没有孔板的方案 基本相同, 基本不产生空泡。吸力面和压力面的空 化现象得到最好的改善。在其他三种方案中, 少量 空泡存在于诱导轮的吸力面。最严重的是传统的 $0^{\circ} / 0^{\circ}$ 型。空泡分布范围最大, 空化最严重。 


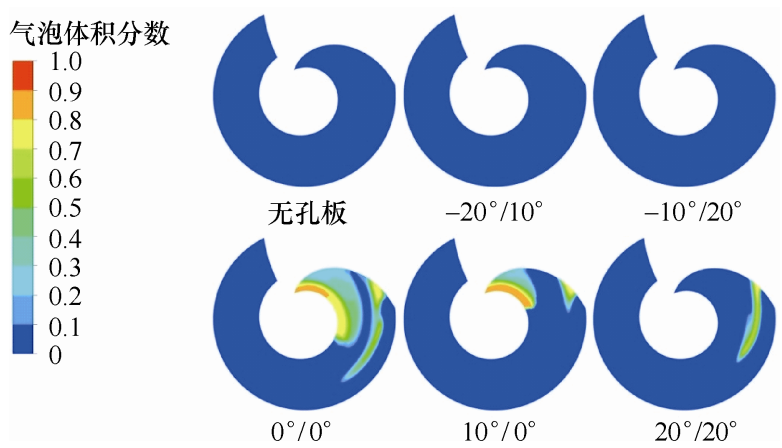

图 11 诱导轮压力面汽相体积分数分布图

为研究分析流道之间的空化现象, 在诱导轮内 部流道的 $1 / 2$ 处截取一截面, 其处的气相体积分布
如图 12 所示。发现在不加孔板的情况下, 汽相体积 分数主要集中分布在诱导轮吸力面上, 并且吸力面 上的汽相体积分数强度较大, 因此空化比较严重; 相比与其他几个方案, 方案 $-20^{\circ} / 10^{\circ}$ 和 $-10^{\circ} / 20^{\circ}$ 中气相体积分数的分布区域最小, 并主要集中在叶 片吸力面靠近进口处周围的流道部分。 $0^{\circ} / 0^{\circ}$ 和 $10^{\circ} / 0^{\circ}$ 方案的强度在几种方案中最小, 但是气相 体积分数的分布范围相对较大; 相比与其他几个 方案, $20^{\circ} / 20^{\circ}$ 方案控制效果最差, 且流道中气 相体积分数的分布区域最广, 强度相对较大, 空 化严重。
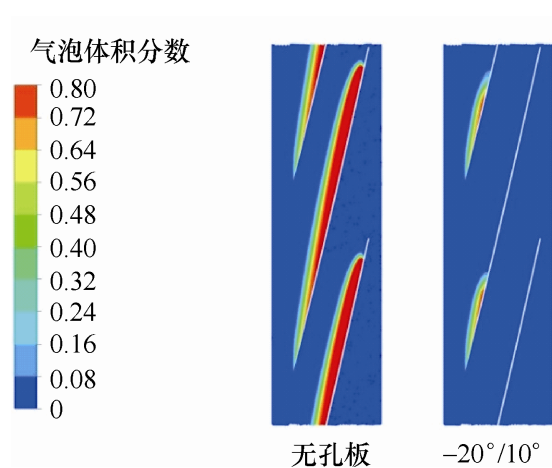

$-20^{\circ} / 10^{\circ}$

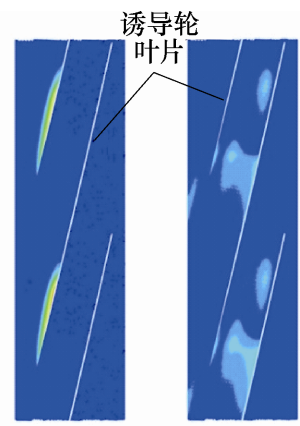

$-10^{\circ} / 20^{\circ}$



$10^{\circ} / 0^{\circ}$

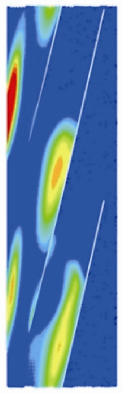

$20^{\circ} / 20^{\circ}$

图 12 诱导轮流道内汽相体积分数分布图

\section{2 叶轮空化特性分析}

在叶轮的内部截取一个平面对叶轮流道内的空化 情况进行观察分析。流道取叶轮中间的截面, 如图 13 所示, 在无孔板的条件下流道内的空化十分严重, 空泡 分布在各个流道内, 强度达到了 0.4 以上, 强度大。在 增加了空化抑制装置孔板之后, 各流道内的汽相体积分
数有着明显的变化, 各方案中叶轮流道内几乎没有空化 产生, 流道内十分顺畅没有空泡, 这与前述分析叶片汽 相体积分数分布情况大体相同, 其中只有 $20^{\circ} / 20^{\circ}$ 这 个情况在叶片的头部分布着非常小的空泡, 这也与在单 相分析时处在叶片头部的前缘处得低压区域相一致, 但 其空泡分布范围不是很大, 影响并不是很大。

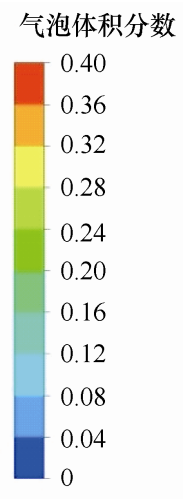

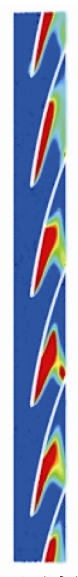

无孔板

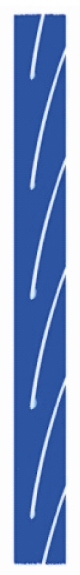

$-20^{\circ} / 10^{\circ}$

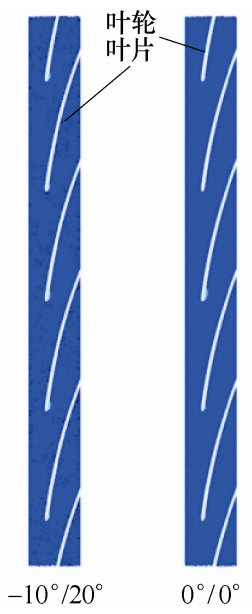

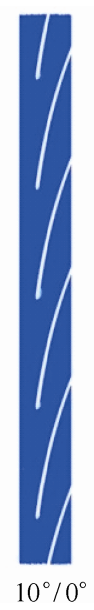

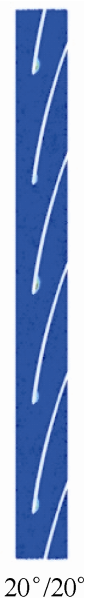

$20^{\circ} / 20^{\circ}$

空化系数和扬程系数计算公式分别为

$$
\begin{gathered}
\sigma=\frac{2\left(P_{1}-P_{v}\right)}{\rho U^{2}} \\
\psi=\frac{2 g H}{D_{2} \pi^{2} n^{2}}
\end{gathered}
$$

式中, $P_{1}$ 为进口压力, $\mathrm{Pa} ; P_{v}$ 为蒸汽的饱和气化压 力, $\mathrm{Pa} ; U$ 为泵出口圆周速度, $\mathrm{m} / \mathrm{s} ; \rho$ 为清水的密 度, $\mathrm{kg} / \mathrm{m}^{3}$ 。

得到无空化抑制装置以及增加抑制装置的各个 方案下的空化外特性曲线, 如图 14 所示。各个工况 


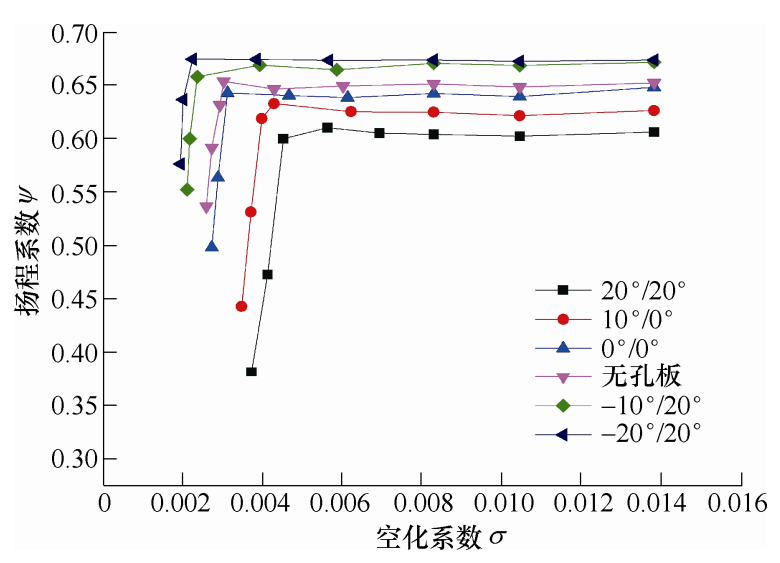

图 14 空化外特性曲线

下的空化外特性曲线趋势大致相同, 曲线刚开始时 接近水平, 随着空化系数的减小到一定数值时, 曲 线发生了陡降, 陡降前出现轻微的波动, 这是由于 叶轮内部的压力不对称引起的。

图 7 中可知, 其中 $-20^{\circ} / 10^{\circ}$ 孔板方案的离心原 扬程系数最高, 其抗汽蚀性能最好。随着孔板的 $\alpha$ 越大, 空化特性曲线陡降所对应的位置越靠前, 空 化系数越小, 离心原的抗汽蚀性能越差。这与上文 中分析高速泵内部汽相体积分数的结果是一致的。

\section{3 高速洜性能分析}

分析倾角不同的孔板对高速离心洜性能的影 响, 计算倾角不同的孔板的扬程和效率, 计算公式 如下。

扬程

$$
H=\frac{p_{\text {out }}-p_{\text {in }}}{\rho g}+\Delta z
$$

轴功率

$$
P=\frac{T \cdot n}{9550}
$$

效率

$$
\eta=\frac{\rho g Q H}{P}
$$

式中, $p_{\text {out }} 、 p_{\text {in }}$ 为离心原的出口与进口的总压力; $\Delta z$ 离心㫤进口和出口之间的高度差; $T$ 为作用在诱 导轮和叶轮上的总力矩; $n$ 为高速百的转速; $Q$ 为 流量; $g$ 为重力加速度; $\rho$ 为介质密度。没有考虑 效率损失, 只是计算水力效率。

通过计算得到各个方案情况下的扬程曲线, 如 图 15 所示。在加孔板或者是不加孔板的条件下扬程 变化规律几乎一样, 流量的增加导致扬程逐渐下降。 在 $0.6 Q_{\mathrm{d}}$ 的小流量工况情况下, 扬程增加比较大的 是 $20^{\circ} / 20^{\circ}$ 方案, 相比于其他情况下大约高出 $10 \mathrm{~m}$ 的水头, 相比于不加孔板的情况下高出大约 $22 \mathrm{~m}$ 的 水头; 在设计工况下, 扬程提高最明显的为 $-20^{\circ} /$

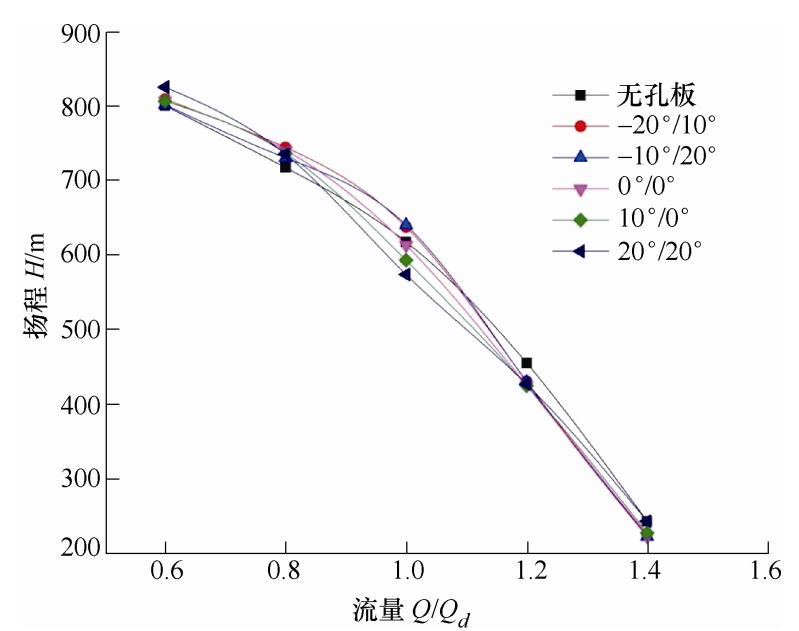

图 15 扬程流量曲线

$10^{\circ}$ 和 $-10^{\circ} / 20^{\circ}$ ，比不加孔板的时候扬程高于 20 $\mathrm{m}$ 左右, $0^{\circ} / 0^{\circ}$ 方案同不加孔板的时候扬程大体相 同, $10^{\circ} 10^{\circ}$ 和 $20^{\circ} / 20^{\circ}$ 的时候扬程都比不加孔板 时的扬程要小; 在 $1.4 Q_{\mathrm{d}}$ 流量工况时, 扬程基本不 变, $20^{\circ} 120^{\circ}$ 方案与不加孔板是的扬程几乎不变, 其他方案的扬程也相差不大, 但是都比不加孔板时 的扬程要小。

再由各方案下效率流量曲线图 16 可知。流量与 效率曲线总体的变化趋势是, 效率随着流量的增大 先增加后减小, 而在设计流量工况时达到最优。在 $0.6 Q_{\mathrm{d}}$ 小流量的工况时, 在有加孔板空化抑制装置时 的效率明显要优于不加孔板时的效率, 而且效率有 很大的提高, 究其原因是由于在数值模拟中液流流 经孔板的各种损失被忽略了, 通过比较分析不同孔 板之间的效率, $0^{\circ} 10^{\circ}$ 方案的效率是最大的, 比其 他方案高出了 1.5 个百分点; 进而在设计工况下, 效率达到了最大, 然而 $-20^{\circ} / 10^{\circ}$ 和 $-10^{\circ} / 20^{\circ}$ 这 两个方案的效率提高最大, 这与之前相分析的情况 基本是相同的, 这两个方案在抑制回流漩浴和控制 泵的空化上都具有很大的优势, 因此对高速泵性能 的提高有极高的参考价值, 传统型孔板 $0^{\circ} / 0^{\circ}$ 方案

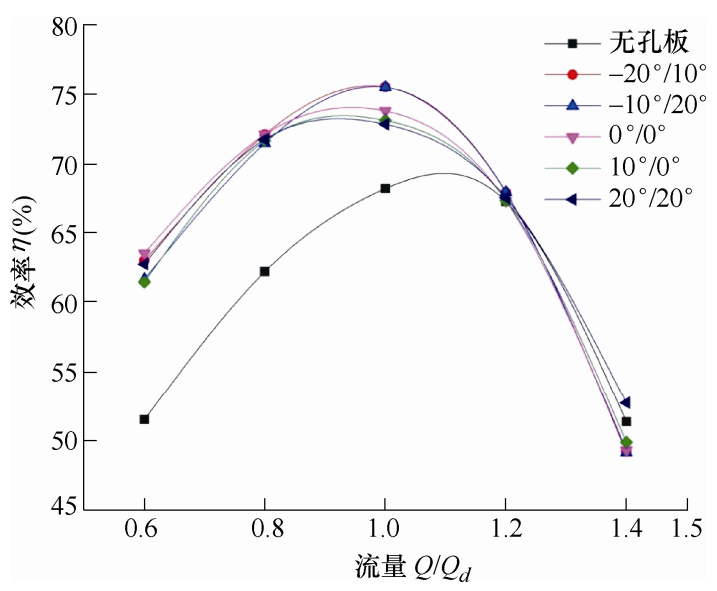

图 16 效率流量曲线 
的效率要比这两个方案大约低于 $2 \%$, 且与其他两 个方案的效率相比, 他们均低于 $0^{\circ} / 0^{\circ}$ 方案; 在 $1.4 Q_{\mathrm{d}}$ 大流量工况下, 其效率最明显提高的是 $20^{\circ} /$ $20^{\circ}$ 方案, 相比无孔板时高出了 $1.4 \%$, 不加孔板方 案的效率都比其他方案的要高。

\section{4 结论}

(1) 从回流漩涡强度和影响范围来看, 对于空 化抑制装置 $-10^{\circ} / 20^{\circ}$ 和 $-20^{\circ} / 10^{\circ}$ 两个方案, 水 流轴向速度全部与主流方向一致, 此时发现在监测 直线 $\mathrm{B}$ 上回流速度为 0 , 不难得出此时方案对回流 漩浴的轴向速度控制效果最佳, 实现了抑制进口回 流的目的。

(2) 在设计工况下, $-20^{\circ} / 10^{\circ}$ 和 $-10^{\circ} / 20^{\circ}$ 两 个方案中诱导轮和叶轮的空化情况都得到了极大的 抑制, 使其性能得到了大大的提升, 叶轮内部的空 化基本消失。从空化外特性曲线可以看出, 其中 $-20^{\circ} / 10^{\circ}$ 孔板方案的离心洜扬程系数最高, 说明 此方案抗空化性能最好, 不难发现空化抑制装置对 高速离心泵内部流动性能有着明显的促进作用。

(3) 在设计工况下, $-20^{\circ} / 10^{\circ}$ 和 $-10^{\circ} / 20^{\circ}$ 两 个方案的扬程较之不加孔板的高速洜扬程提升约 $20 \mathrm{~m}$, 相较传统型孔板 $0^{\circ} 10^{\circ}$ 方案的效率提高大 约 $2 \%$ 。

（4）通过在离心厡, 特别在高速离心泵进口处增 加空化抑制装置, 并对各个方案进行研究, 提出了最 优的空化抑制装置方案为 $-10^{\circ} / 20^{\circ}$ 和 $-20^{\circ} / 10^{\circ}$ 。

\section{参 考 文 献}

[1] 朱祖超, 何月秋, 王晓洁. 低比转速高速离心泵的理论 及设计应用[M]. 北京: 机械工业出版社, 2011.

ZHU Zuchao, HE Yueqiu, WANG Xiaojie. Theory and design application of low specific speed high speed centrifugal pump[M]. Beijing: China Machine Press, 2011.

[2] 魏立超, 宋文武, 石建伟, 等. 基于 CFD 的高速离心 泵进口段流动数值模拟分析 [J]. 热能动力工程, 2016, 31(7): 103-109.

WEI Lichao, SONG Wenwu, SHI Jianwei, et al. Numerical simulation and analysis of the flow at the inlet of a high speed centrifugal pump based on the software CFD $[J]$. Journal of Engineering for Thermal Energy and Power, 2016, 31(7): 103-109.

[3] 袁建平, 周帮伦, 孙威, 等. 离心泵进口回流特性的 CFD 与试验研究 $[\mathrm{J}]$. 中国农村水利水电, 2014(7): $162-166$.
YUAN Jianping, ZHOU Banglun, SUN Wei, et al. Research on the inlet recirculation's characteristics in centrifugal pumps based on CFD and experiment methods[J]. China Rural Water and Hydropower, 2014 (7): $162-166$.

[4] 赵宇, 王国玉, 吴钦, 等. 基于计算流体力学的串列轴 流泵空化性能分析[J]. 机械工程学报, 2014, 50(6): 171-176.

ZHAO Yu, WANG Guoyu, WU Qin, et al. Analysis of Cavitation performances of an axial flow tandem pump based on computational fluid dynamics[J]. Journal of Mechanical Engineering, 2014, 50(6): 171-176.

[5] 高波, 孙金恺, 杨敏官, 等. 离心原内空化流动诱导非 定常激励特性 [J]. 机械工程学报, 2014, 50(16): 199-205.

GAO Bo, SUN Xinkai, YANG Minguan, et al. Characteristics of unsteady excitation induced by cavitating flow in centrifugal pumps[J]. Journal of Mechanical Engineering, 2014， 50(16): 199-205.

[6] 袁寿其, 梁望, 袁建平, 等. 离心泵进口回流流场特性 的数值模拟及试验 [J]. 排灌机械工程学报, 2011, 29(6): 461-465.

YUAN Shouqi, LIANG Yun, YUAN Jianping, et al. Numerical simulation and experiment on characteristics of centrifugal pump inlet recirculation[J]. Journal of Drainage and Irrigation Machinery Engineering, 2011, 29(6): 461-465.

[7] 黄建德. 离心泵进口回流的发生机理及预估 [J]. 上海 交通大学学报, 1998, 32(7): 5-9.

HUANG Jiande. Mechanism and prediction of inlet reverse flow onset in centrifugal pump impellers[J]. Journal of Shanghai Jiaotong University, 1998, 32(7): 5-9.

[8] QIU N, L WANG Q, KONG F Y, et al. Research on cavitation characteristic of inducer[J]. IOP Conference Series: Materials Science and Engineering, 2013，52(6): 2010.

[9] KIRIS C C, KWAK D, CHAN W, et al. High-fidelity simulations of unsteady flow through turbo pumps and flowliners[J]. Computers \& Fluids, 2008, 37(5): 536-546.

[10] ITO Y, TSUNODA A, NAGASAKI T. Experimental comparison of backflow-vortex cavitation on pump inducer between cryogen and water[J]. Journal of Physics: Conference Series, 2015, 656(1): 012063.

[11] YAMANISHI N, FUKAO S, QIAO X, et al. LES simulation of backflow vortex structure at the inlet of an inducer[J]. Journal of Fluids Engineering, 2007, 129(5): 587-594.

[12] SI Q, YUAN S, YUAN J, et al. Investigation on flow-induced noise due to backflow in low specificspeed centrifugal pumps[J]. Advances in Mechanical Engineering, 
2013， 5: 109048.

[13] SINGHAL A K, ATHAVALE M. Mathematical basis and validation of the full cavitation model[J]. Journal of Fluids Engineering, 2002, 124: 617-624.

[14] ADRIAN Stuparu, ROMEO Susan-Resiga, LIVIU Eugen Anton, et al. A new approach in numerical assessment of the cavitation behaviour of centrifugal pumps[J]. International Journal of Fluid Machinery and Systems, 2011(4): 104-113.

[15] RAFAEL C A, FARID B, ALFONSO C A, et al. Numerical analysis of unsteady cavitating flow in an axial inducer[J]. Applied Thermal Engineering, 2015(75): 1302-1310.

[16] FRASER W H. Flow recirculation in centrifugal pumps[J]. World Pumps, 1981, 132(8): 95-100.

[17] OSHIMA M. A study on suction performance of a centrifugal pump with an inducer[J]. Bulletin of JSME, 1967, 10(42): 959-965.

[18] 郭晓梅, 朱祖超, 崔宝玲, 等. 基于正交试验的诱导轮 前置孔板的参数化研究 [J]. 工程热物理学报, 2011 , 32(8): 1319-1322.

GUO Xiaomei, ZHU Zuchao, CUI Baoling, et al. Study of pre-positioned orifice's parameters before the inducer based on orthogonal test[J]. Journal of Engineering Thermophysics, 2011, 32(8): 1319-1322.

[19] 付燕霞, 沈陈栋, 袁建平, 等. 离心泵进口回流诱导的 空化特性[J]. 排灌机械工程学报, 2016(10)：841-846. FU Yanxia, SHEN Chendong, YUAN Jianping, et al. Cavitation characteristic induced by inlet backflow in a centrifugal pump[J]. Journal of Drainage and Irrigation Machinery Engineering, 2016 (10): 841-846.

[20] 张金风, 梁望, 袁建平, 等. 离心泵进口回流流场及其 控制方法的数值模拟 [J]. 江苏大学学报, 2012, 33(4): 402-407.

ZHANG Jinfeng, LIANG Yun, YUAN Jianping, et al. Numerical simulation of recirculation control at centrifugal pump inlet[J]. Journal of Jiangsu University 2012，33(4): 402-407.

作者简介: 宋文武, 男, 1965 年出生, 教授, 博士研究生导师。主要从 事流体机械研究。

E-mail: wenwus@163.com

石建伟(通信作者), 男, 1988 年出生, 讲师。主要从事流体机械内部流动及 新产品的开发研究。

E-mail: shijianwei0416@163.com 